\title{
Inferior Vena Cava Filters
}

\author{
Thomas B. Kinney, M.D., M.S.M.E. ${ }^{1}$
}

ABSTRACT

Venous thromboembolism (VTE) remains a common disease with significant clinical impact upon our patients. Diagnostic challenges occur because of the nonspecific nature of the presenting symptoms. The advent of multidetector computed tomography, methods to stratify patients into VTE risks (low, intermediate, high) along with serological assays (D-dimers), have helped direct patients through proper workup and into conclusive diagnosis. In most cases, standard medical therapy for VTE is anticoagulation therapy (OAT). In situations where standard OAT is either contraindicated or complications result from that therapy, insertion of inferior vena cava (IVC) filters is considered. Recent reports suggest that although IVC filters are able to prevent pulmonary emboli $(\mathrm{PE})$ in the short and intermediate term, there appear to be long-term consequences including excess recurrent deep venous thombosis (DVT and IVC/filter occlusions). Recognition of the time sequence of IVC filter benefits and complications has encouraged development of optional IVC filters, which can be left in place indefinitely or removed usually before certain time constraints. This article will attempt to address the timing of IVC filter placements to protect patients from significant PE.

KEYWORDS: Venous, thrombosis, embolism, pulmonary, venae cavai, filters

Objectives: Upon completion of this article, the reader should be able to (1) identify the various indications for insertion of IVC filters (absolute, relative, prophylactic), (2) review the long-term functional aspects of IVC filters, and (3) review the timing of IVC filter insertions (emergent, urgent, and elective).

Accreditation: Tufts University School of Medicine (TUSM) is accredited by the Accreditation Council for Continuing Medical Education to provide continuing medical education for physicians.

Credit: TUSM designates this educational activity for a maximum of 1 Category 1 credit toward the AMA Physicians Recognition Award. Each physician should claim only those credits that he/she actually spent in the activity.

Venous thromboembolism (VTE) represents a spectrum of disease that extends from deep venous thombosis (DVT) to pulmonary emboli (PE). VTE occurs for the first time in $\sim 100$ persons per 100,000/year in the United States; the rate rises exponentially with age, from $<5$ cases per 100,000 in patients $<15$ years old to $\sim 500(0.5 \%)$ per 100,000 persons at age 80 years. $^{1}$ Approximately one-third of patients with symptomatic VTE present with PE, and the remaining two-thirds manifest with DVT alone. Despite anticoagulation

${ }^{1}$ Professor of Clinical Radiology, UCSD Medical Center, San Diego, California.

Address for correspondence and reprint requests: Thomas B. Kinney, M.D., M.S.M.E., Professor of Clinical Radiology, UCSD Medical Center, 200 West Arbor Drive, San Diego, CA 921038756. therapy, VTE recurs frequently in the first few months after the proximal event, with a recurrence rate of $\sim 7 \%$ at 6 months. Death occurs in $\sim 6 \%$ of DVT cases and $12 \%$ of PE cases usually within 1 month of diagnosis.

The medical treatment of $\mathrm{PE}$ was revolutionized by the use of heparin. ${ }^{2}$ OAT is associated with a small $(<5 \%)$ risk of major hemorrhage, but increased bleeding risks do occur in patients with thrombocytopenias, central nervous neoplasms including metastases, or active gastrointestinal bleeding. ${ }^{3}$ In these patients and
Interventional Radiology On-Call; Guest Editor, Thuong G. Van Ha, M.D.

Semin Intervent Radiol 2006;23:230-239. Copyright (C) 2006 by Thieme Medical Publishers, Inc., 333 Seventh Avenue, New York, NY 10001, USA. Tel: +1(212) 584-4662.

DOI 10.1055/s-2006-948760. ISSN 0739-9529. 
Table 1 FDA-Approved IVC Filters for Use in the United States

\begin{tabular}{|c|c|c|c|c|c|c|}
\hline Filter & $\begin{array}{l}\text { Sheath } \\
\text { (French) }\end{array}$ & $\begin{array}{l}\text { Max Caval } \\
\text { Diameter (mm) }\end{array}$ & $\begin{array}{l}\text { Guide Wire } \\
\text { Delivery }\end{array}$ & Access & Retrievable & Material \\
\hline Titanium Greenfield $^{\dagger}$ & 14.3 OD & 28 & No & $F, J$ & No & Titanium \\
\hline $\begin{array}{l}\text { 12-F Stainless Steel } \\
\text { O-T-W Greenfield }{ }^{\dagger}\end{array}$ & $15.6 \mathrm{OD}$ & 28 & Yes & $F, J$ & No & Stainless steel \\
\hline Vena Tech LGM ${ }^{\ddagger}$ & $14.6 \mathrm{OD}$ & 28 & No & $F, J$ & No & Phynox \\
\hline Simon Nitinol" & 9 OD & $\begin{array}{c}28 \text { (24 if surgery or } \\
\text { manipulation*) }\end{array}$ & No & $F, J, A$ & No & Nitinol \\
\hline Recovery" & $7 \mathrm{OD}$ & 28 & No & $\mathrm{F}$ & Yes & Nitinol \\
\hline Vena Tech LPł & 6 & 30 & No & $F, J$ & No & Phynox \\
\hline TrapEase & 6 & 30 & No & $F, J, A$ & No & Nitinol \\
\hline OptEase & 6 & 30 & No & $F, J, A$ & Yes & Nitinol \\
\hline
\end{tabular}

*Within 2 weeks of implantation.

†Boston Scientific, Natick, MA

B. Braun Medical, Allentown, PA.

${ }^{\S}$ Cook, Inc., Bloomington, IN.

"Bard Peripheral Vascular, Tempe, AZ.

'Cordis Corporation (J\&J), Miami, FL.

FDA, Food and Drug Administration; F, femoral; J, jugular; A, arm; OD, outer diameter.

many others who cannot undergo anticoagulation for a diversity of reasons, interruption of the inferior vena cava (IVC) is considered.

Although it is now well understood that lower limb DVT has been found to be responsible for over $90 \%$ of $\mathrm{PE}$, the lower extremity DVT is apparent in only $10 \%$ of patients. In general, the culprit veins for the $\mathrm{PE}$ involve the larger veins downstream from the trifurcation (popliteal vein and above). Symptoms of PE ensue only when a large embolus occurs and only those greater than $7.5 \mathrm{~mm}$ in diameter are likely to be fatal, although preexisting cardiopulmonary compromise increases a patient's vulnerability to embolic insults. Other rarer causes of PE may include DVTs in iliac, renal, and hepatic veins; the right heart; and the upper extremity veins especially patients with catheters or pacemakers.

\section{HISTORICAL PERSPECTIVE ON IVC INTERRUPTION}

The historical aspects of IVC interruption are fascinating, including many prominent innovators, procedures, and devices ${ }^{4,5}$ but these cannot be presented here because of length constraints. The current generation of IVC filter devices derives much from the design implementation and performance characteristics obtained from the Greenfield IVC filter, which was introduced in $1973 .{ }^{6}$ This filter is conical in shape with the narrower section placed downstream in the cava. The conical shape provides geometric clot packing efficiency. A large length of the filter (up to 70\%) can be filled with clot while still providing patency to half of the cross-sectional area of the vena cava. The filter-trapped clot lies within the central flow steams of the cava allowing para-axial flow. Maintenance of caval flow delivers endogenous fibrinolytic agents along with mechanical fragmentation of clots based upon flow pressures. Theoretically, these two favors help maintain cava patency while reducing significant PE. Preventing significant PE and maintaining caval patency are two key functional attributed of these devices. Since the introduction of this filter several other filter designs, now all percutaneously placed, have been developed. Most filters utilize the original conical shape or deviations on this, although others clearly use vastly different methods of clot capture (Table 1). Three of the filters can be used either for permanent implantation or can be removed after various indwell periods (optional IVC filters).

\section{Desirable Attributes of IVC Filters}

Several IVC filter attributes have been recognized as having desirable characteristics, recognizing that

Table 2 Desirable Attributes of an "Ideal IVC Filter",4,7

Nonthrombogenic, biocompatible, infinite implant life

High filter efficiency (large and small emboli) with no resistance to flow

Secure fixation within the IVC with no migration or tilting

Ease of percutaneous insertion

Small delivery system

Easy to use, controlled release mechanism

Potentially amenable to repositioning

Magnetic resonance imaging compatible

Low cost

Low access site thrombosis

Ease and efficacy of retrievability 
achievement of some attributes may occur at the determent of others (Table 2).

\section{CONTROVERSIAL ASPECTS ABOUT IVC FILTERS}

Insertion of IVC filters is offered to protect patients from life-threatening $\mathrm{PE}$ while subjecting them to limited, but potentially significant infrequent complications. Although most interventional radiologists readily accept this risk-benefit ratio for IVC filter insertion for patients with document VTE who cannot be on anticoagulation medicine, there are many controversial aspects about IVC filters that are emphasized by our pulmonary and hematological colleagues. ${ }^{7-10}$ Unfortunately, numerous early studies used to answer these difficult questions were historical case series studies with many limitations. Recently, the PREPIC study group conducted an open, randomized, multicenter study of anticoagulation plus vena cava filter placement in 400 patients with acute proximal DVT. ${ }^{11}$ Four different filters were used: the Vena Tech filter (56\%), the titanium Greenfield filter (26\%), the Cardial filter (Bard, Saint-Etienne, France) (not available in the United States), and the Bird's Nest Filter IVC filter (15.5\%). All patients had nuclear medicine V/Q scans or pulmonary angiography at baseline or between days 8 and 12. At 12-day follow-up, there was a significant difference in the number of PEs, favoring the filter group. In the filter group, there were two PEs (1.1\%), both symptomatic, versus nine PEs (4.8\%; $p=0.03)$ in the nonfilter group. Of these nine PEs in the nonfilter group, five were symptomatic and four were asymptomatic. However, by 2 years this statistical advantage for IVC filters disappeared. In the filter group, six PEs (3.4\%) were found compared with 12 PEs $(6.3 \% ; p=0.16)$ found in the nonfilter group. Also at 2 years the filter group had a significant increase in recurrent DVT (37 DVT; 20.8\%) compared with 21 DVT $(11.6 \% ; p=0.02)$ in the nonfilter group. These data indicate that IVC filters provide significant additional short-term protection from PE compared with anticoagulation alone, which wanes over time and is associated with increased risk of recurrent DVT. A significant number of the patients with recurrent DVT in the filter arm of the study also had IVC filter thromboses. More recently, 8-year follow-up data of this study have become available. ${ }^{12}$ At 8 years, PE occurred in nine patients with filters (cumulative rate of $6.2 \%)$ and 24 patients (15.1\%) in the nonfilter group $(p=0.042)$. Postthrombotic syndrome was seen similarly in both groups. The authors concluded that "at 8 years, vena cava filters reduced the risk of pulmonary embolism but increased that of deep-vein thrombosis and had no effect on survival. Although their use may be beneficial in patients at high risk of pulmonary embolism, systematic use in the general population with venous thromboembolism is not recommended." Note that this study used IVC filters in situations not commonly seen on our practice.

\section{PATHOPHYSIOLOGY OF VENOUS THROMBOEMBOLISM}

The pathophysiology of DVT was elucidated by Virchow who hypothesized a triad of factors involved, which included venous intimal injury, stasis, and hypercoagulability. Although questions remain about the precise relationship between DVT and PE, historical studies suggest that DVT almost always precedes PE and PE most often occurs in the setting of DVT, which in most cases is asymptomatic. ${ }^{13}$ Acute DVT is loosely attached to the venous endothelium and may propagate to the venous wall and/or embolize (PE). By 7 to 10 days, this thrombus starts to adhere to the vein wall and inflammatory changes start occurring. Neovascularization and fibroblast infiltration of thrombus causes scarring and damages the venous valves, which become incompetent, allowing venous reflux and venous hypertension. Indeed, in the orthopedic surgical literature the highest risk of $\mathrm{PE}$ occurs relatively early with a peak incidence of $\mathrm{PE}$ occurring between the first and second week after hip replacement and 7 days following knee replacement surgery. ${ }^{14}$ Most symptomatic PEs arise in the deep veins of the thigh. Deep veins of the calf, upper extremity, and subclavian and jugular systems are less likely to result in clinically significant PE.

\section{CONVENTIONAL TREATMENT OF VTE}

The treatment of VTE was revolutionized by the 1960 Barritt and Jordan report on a placebo-controlled trial used to justify heparin and vitamin $\mathrm{K}$ antagonists as treatment for DVT. ${ }^{15}$ The study actually consisted of 39 patients clinically diagnosed with PE (the DVT patient subpopulation was not reported). The clinical diagnoses were not confirmed with pulmonary angiography nor with nuclear medicine lung scans. The small study noted that patients who had survived symptomatic PE and received anticoagulants had significantly lower mortality from $\mathrm{PE}(0 / 16$ with anticoagulants compared with 5/19 [26.3\%] with placebo [ $p<0.0007])$. No recurrent PEs occurred in the anticoagulated patients, although 10/19 (53\%) of the patients treated with placebo had recurrent PEs. Although controversial issues still remain, in general a first course of unfractionated or low-molecularweight heparin (LMWH) followed by at least 3 months of oral anticoagulant therapy (OAT) is the treatment of choice in patients with acute VTE episodes. ${ }^{16}$ The OAT aims to prevent death after PE, stop DVT extension, and reduce the rate of recurrent VTE. In a meta-analysis of VTE patients treated with OAT, the rate of fatal PE 
among patients resenting with DVT was $0.4 \%$, and the rate of fatal $\mathrm{PE}$ for patients presenting with $\mathrm{PE}$ was $1.5 \%{ }^{17}$ Survival after VTE is worse than expected, and survival after $\mathrm{PE}$ is much worse than after DVT. ${ }^{18}$ The risk of early death among patients with symptomatic $\mathrm{PE}$ is 18-fold higher compared with patients with DVT alone. $\mathrm{PE}$ is an independent predictor of reduced survival for up to 3 months after onset. For $\sim 25 \%$ of patients, the initial clinical presentation is sudden death. Some of the independent predictors of reduced early survival after VTE include advancing age, male gender, reduced body mass index, occurrence of VT while in a hospital of nursing home, congestive heart failure, chronic lung disease, serious neurological disease, and active malignancy. Other predictors of poor early survival after PE include syncope and arterial hypotension. Evidence of right heart dysfunction based upon clinical exam, laboratory markers, or echocardiography predicts poor survival even among normtensive PE patients. $\mathrm{PE}$ patients with these characteristics warrant aggressive anticoagulation therapy and consideration for thrombolytic therapy either systemically or delivered via catheter directly to the pulmonary circulation. Adjunctive percutaneous mechanical techniques to break down thrombus within the pulmonary circulation have also been described.

After a first episode of VTE, the risk of recurrence is relatively high with potentially serious clinical consequences, as acute (in 5\% of patients) and chronic (postthrombotic syndrome) complications are frequent. ${ }^{17}$ VTE recurs in $\sim 30 \%$ of patients within 10 years, with the risk being highest within the first 6 to 12 months. Although the incident event type (DVT alone versus $\mathrm{PE}$ ) is not a predictor of recurrence, patients with recurrence are significantly more likely to recur with the same event type as initially. The 7-day case fatality rate is significantly higher for recurrent $\mathrm{PE}$ (34\%) than with DVT alone (4\%). The amount of time that subjects with an acute unprovoked VTE event spend at near-normal INR values $(<1.5)$ during the first 3 months of treatment is associated with higher VTE recurrence. ${ }^{19}$

\section{INDICATIONS FOR IVC FILTER INSERTION}

It is prudent to stick with strict criteria when asked to evaluate a patient for potential IVC filter insertion. Generally, indications for all vena cava filters have been broadly broken down into absolute, relative, prophylactic categories (Table 3). ${ }^{4}$ At the present time, placement of optional filters with the intent to discontinue filtration through either retrieval or conversion follow the same indications as those used for permanent IVC filters. ${ }^{20}$ It must be emphasized that although an IVC filter will effectively reduce the frequency of significant $\mathrm{PE}$, it has no affect whatever on the underlying thrombotic process and may exacerbate this (see above).
Table 3 Indications for IVC Filter Insertion ${ }^{4}$

Absolute indications (proven VTE)
Recurrent VTE (acute or chronic) despite adequate
anticoagulation
Contraindication to anticoagulation
Complication of anticoagulation
Inability to achieve/maintain therapeutic anticoagulation
Relative indications (proven VTE)
Iliocaval DVT
Large, free-floating proximal DVT
Difficulty establishing therapeutic anticoagulation
Massive PE treated with thrombolysis/thrombectomy
Chronic PE treated with thromboendarterectomy
Thrombolysis for iliocaval DVT
VTE with limited cardiopulmonary reserve
Recurrent PE with filter in place
Poor compliance with anticoagulation medications
High risk of complications of anticoagulation (e.g., ataxia,
frequent falls)
Prophylactic indications (no VTE, primary prophylaxis is not
feasible)
Trauma patient with high risk of VTE
Surgical procedure in patient with high risk of VTE
Medical condition with high risk of VTE
Contraindication to IVC filter
No access to the vena cava
No location available in vena cava for placement of a filter

\section{TIMING OF VENOUS INTERRUPTION}

There is not much useful literature guiding the physician as to the timing of insertion of IVC filters once a request for filtration is made. The risk-benefit ratio for filter placement as well as urgency of insertion varies with each indication (Tables 3 and 4). Jones et al proposed guidelines and introduced three different categories based upon urgency of the clinical situation. ${ }^{21}$

\section{Emergent Filter Placement/Intervention}

Emergent placement, generally within the first 12 to 24 hours, is indicated in patients with acute PE or freefloating iliofemoral DVT in whom OAT is contraindicated or has failed and in patients with severe cardiopulmonary reserve who are felt to be unable to tolerate any embolic insults. Contraindications to OAT include active or recent neurological, pulmonary, gastrointestinal, or urologic bleeding while on anticoagulation; heparin-associated thrombocytopenia thrombosis syndrome; recent major trauma or surgery. In general, these patients are treated emergently as they are unprotected from potentially life-threatening PE. Using the Barritt data (above) the mortality of recurrent $\mathrm{PE}$ in such patients may be as high as $26 \%$, and in almost one-third of these patients the lethal recurrence will occur within 24 hours of the initial event. $^{22}$ Patients in whom symptomatic DVT initially 
Table 4 Indications and Timing for Placement of IVC Filters

\begin{tabular}{|c|c|c|c|}
\hline \multirow[b]{2}{*}{$\begin{array}{l}\text { Anticoagulation } \\
\text { Status }\end{array}$} & \multicolumn{3}{|c|}{ Clinical Circumstance* } \\
\hline & PE & DVT & $\begin{array}{l}\text { High Risk for } \\
\text { PE or DVT }\end{array}$ \\
\hline Contraindicated & Emergent & Urgent $^{\dagger}$ & Elective $^{\ddagger}$ \\
\hline Failed & Emergent & Urgent $^{\dagger}$ & Variable \\
\hline \multirow[t]{3}{*}{ Inadequate } & Emergent & Emergent & $\begin{array}{c}\text { Emergent/ } \\
\text { urgent }^{5}\end{array}$ \\
\hline & \multicolumn{3}{|c|}{ Sources of Recurrent PE } \\
\hline & IVC & & SVC \\
\hline $\begin{array}{l}\text { Mechanical } \\
\text { device failure }\end{array}$ & Emergent & & Investigational \\
\hline
\end{tabular}

*Emergent means the procedure should be done within 24 hours: urgent means it should be done at next available interventional or operating room date; elective means the procedure can be scheduled normally.

Except for free-floating iliofemoral thrombus, which requires emergent filter placement.

The high risk for PE/DVT category with contraindication to anticoagulation may contain the high-risk trauma patients. The timing here is emergent to urgent.

In the original form this category was investigational and included high risk for DVT/PE and trauma patients who were felt to be inadequately treated with anticoagulation. The Eastern Association for the Surgery of Trauma (EAST) evidenced-based guidelines have been added. ${ }^{37}$

PE, pulmonary embolism; DVT, deep venous thrombosis; IVC, inferior vena cava; SVC, superior vena cava.

Data adapted from Jones et al. ${ }^{21}$

develops without $\mathrm{PE}$ who are given only supportive treatment have a 6 to $14 \%$ chance of sustaining a lethal PE. ${ }^{22}$ The risk in the latter cases depends upon the location, type, and extent of thrombus with three-fourths or more of lethal emboli expected to arise in the iliofemoral region.

Some studies suggest a very high incidence of $\mathrm{PE}$ associated with free-floating iliofemoral thrombus despite OAT. In one retrospective study involving 78 patients with venographically proven iliofemoral DVT, the detection of symptomatic PE confirmed by highprobability radioisotope ventilation-perfusion lung scanning within 10 days after venography was 9\% (7/78) despite conventional OAT. ${ }^{23}$ The proximal intraluminal thrombus was characterized as free-floating $(>5-\mathrm{cm}$ nonadherent segment) or occlusive (no free-floating elements). When subclassified by free-floating or occlusive iliofemoral DVT, the incidence of PE diagnosed within 10 days on lung scans was $60 \%(3 / 5)$ compared with $5.5 \%(4 / 73 ; p<0.05)$, respectively (Fig. 1). Despite this data, other studies suggest free-floating thrombus has no higher risk for PE. ${ }^{8}$ One prospective study included 90 patients with 28 patients having occlusive thrombus and 62 patients having free-floating thrombus. ${ }^{24}$ Venography, color venous duplex scanning, and perfusion lung scans were done on admission. If the perfusion scans were abnormal, pulmonary angiography was done within 24 hours. The perfusion lung scan was repeated on days 9 to 11 . The two groups were well

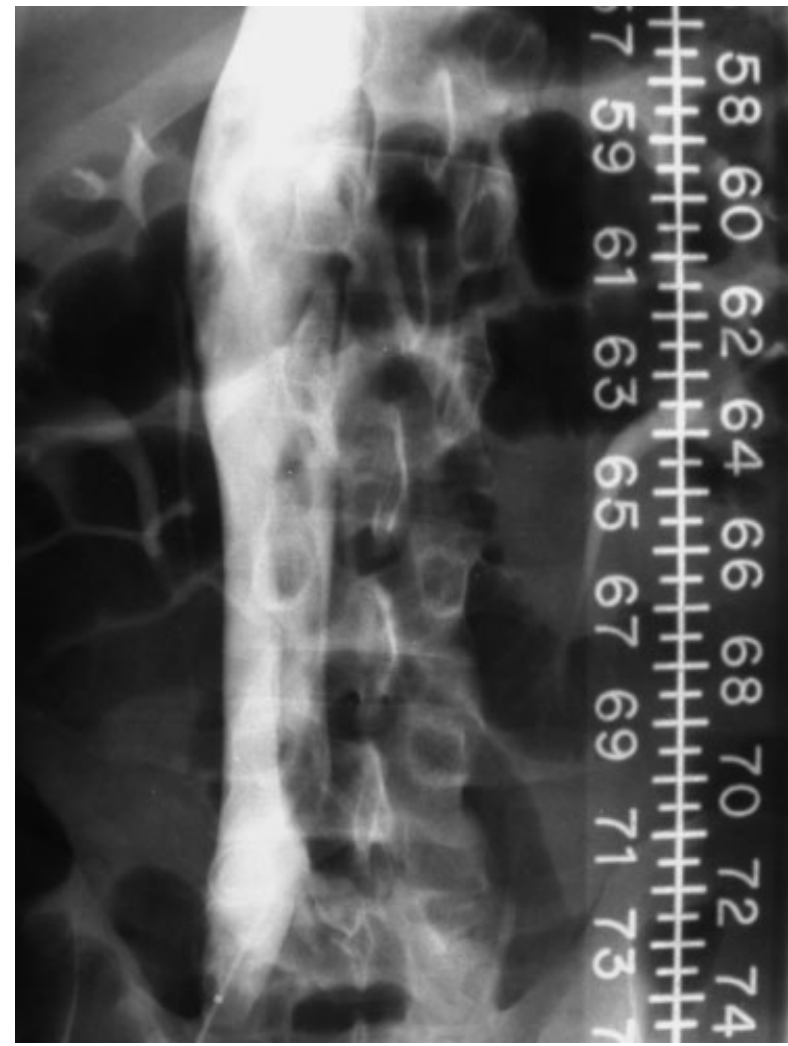

Figure 1 A 22-year-old male patient with ulcerative colitis who developed a spontaneous left lower-extremity DVT. He was treated with OAT and developed hematochezia. Because of this and upcoming planned colectomy, his surgeons requested an IVC filter. The cavagram was performed from the right common femoral vein and shows a large free-floating iliocaval thrombus. Using a right internal jugular approach a suprarenal Greenfield IVC filter was placed and the patient underwent uneventful colectomy. The risk of PE in such settings despite OAT is somewhat controversial.

matched except with clot location: there were 42 iliofemoral clots in the 62 patients with occlusive thrombus in comparison with 23 iliofemoral clots in the 28 patients with free-floating thrombus. The number of patients with $\mathrm{PE}$ on day 10 despite OAT was two versus one patient in those patients with free-floating thrombus compared with occlusive thrombus patients, respectively (NS, $p=0.92)$.

Anticoagulation failure is considered in the following situations: documented recurrent $\mathrm{PE}$, progressive DVT, or complication requiring discontinuation of therapy. In the minority of cases, recurrent VTE occurs despite adequate conventional anticoagulation. A more common scenario includes anticoagulation failures from poor patient compliance, improper dosage, or inadequate monitoring of coagulation status. Furthermore, important drug interactions between warfarin and azole antibiotics, macrolides, quinolones, nonsteroidal antiinflammatory drugs, selective serotonin reuptake inhibitors, omeprazole, lipid-lowering agents, amiodarone, and fluorouracil suggest that coadministration should be avoided or closely monitored. The latter situation may 
result in dangerous under- or overanticoagulation. Note if a reason for failure of anticoagulation is identified that can be readily rectified this may be all that is required to protect the patient from recurrent VTE. However, in situations that are not readily elucidated or rectified, mechanical protection may be necessary. With each VTE event, the likelihood of another event increases; although the mortality rate in untreated patients who have had a $\mathrm{PE}$ is $30 \%$, this increases to $60 \%$ after a second PE occurs. ${ }^{25}$ Presumably, the mortality associated with each recurrent VTE is also increased in those patients receiving failed anticoagulation; consequently additional mechanical protection is indicated by placement of an IVC filter emergently, generally within 24 hours.

Rarely, a patient who has experienced a VTE event cannot receive adequate anticoagulation from standard doses of medication and this is considered inadequate anticoagulation. Often times, these patients need hematologic evaluation to determine the cause of this resistance, and if no anticoagulation method can be used in the high-risk situation, then an IVC filter should be inserted.

In addition, there are a few specific subsets of patients who may be subjected to a high incidence of lethal PE despite conventional OAT. These subsets of patients are often managed by anticoagulation to treat the initiating thrombotic event or tendency along with IVC filtration to protect against an unacceptably high incidence of lethal PE. One example would be a patient who sustained a massive $\mathrm{PE}$ requiring surgical or percutaneous embolectomy/thrombolysis who is at high risk of an immediate recurrence despite anticoagulation. Greenfield and colleagues reported that 2 of $8(25 \%)$ initial survivors of suction embolectomy died of recurrent $\mathrm{PE}$ within 6 hours of the procedure and the current management adds IVC filter placement at completion of all embolectomy procedures. ${ }^{26}$ In a small subset of patients with severe pulmonary hypertension or cor pulmonale, the patients' ability to tolerate any additional embolic insults may be limited. Even though clinically significant recurrent $\mathrm{PE}$ is rare after anticoagulation therapy, carefully controlled studies reveal that the incidence of recurrent PE may be as high as $23 \%$ shortly after the initiation of heparin therapy. ${ }^{27}$ Therefore, the added protection of IVC filters is suggested in such patients. ${ }^{28}$ Patients with septic thrombophlebitis usually require anticoagulation, antibiotic therapy, and drainage to be managed properly. When there is delay in controlling of the peripheral septic site, overwhelming pulmonary sepsis can occur because of multiple septic PE. Although somewhat controversial, IVC filter insertion has been suggested to minimize the associated life-threatening pulmonary complications. ${ }^{29,30} \mathrm{It}$ is hypothesized that the inert stainless steel of the Greenfield filter would trap septic emboli without perpetuating the infection and would allow appropriate antibiotics to sterilize the septic emboli. Some investigators have urged that retrievable IVC filters be used in these situations so that they can be removed if the infection cannot be resolved. . $^{31,32}$

All the mechanical devices used to prevent $\mathrm{PE}$ can fail for multiple reasons. Deployment of the IVC filter offers many potential pitfalls. For instance, a filter may be deployed in a hepatic, renal, iliac, or gonadal vein and may appear to be properly positioned on anteroposterior venograms. Often in these cases, additional views, venography, and recognition of the unusual configuration, positioning, and lack of opening of the filter may reveal to the operator what is going on. Clearly in these cases, the patient is not adequately protected and an additional filter may be placed more optimally and the wayward IVC filter removed, if possible. Other deployment issues may occur when an IVC filter is deployed in a single moiety of a duplicated IVC (Fig. 2). Careful review of any crosssectional imaging studies the patient may have prior to IVC filters insertion should mitigate this happenstance

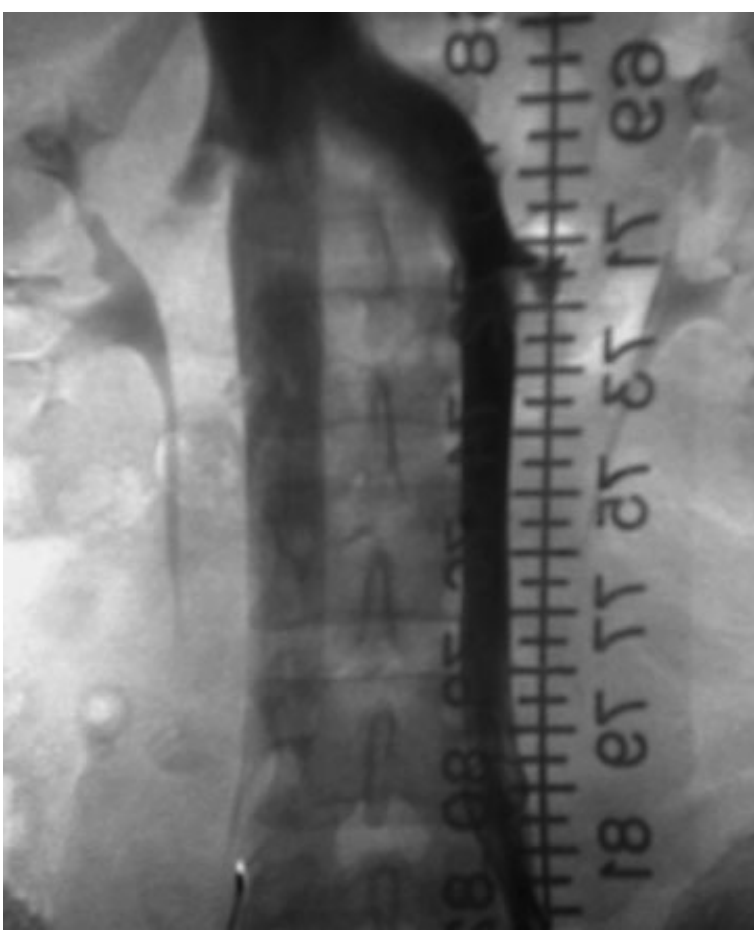

Figure 2 A 25-year-old male who suffered massive trauma including head injury and multiple pelvis and long bone fractures and who was consulted for prophylactic IVC filter placement. An initial cavagram performed from the right common femoral vein showed a patent cava of normal size without thrombus. The left renal inflow into the IVC was noted to be substantially larger than from the right renal vein. A selective left venogram revealed the caval duplication, which is better illustrated by adding a catheter from the left common femoral vein. Two IVC filters (infrarenal) were inserted to completely protect the patient from embolism from a lower extremity source. An alternative approach could include a single suprarenal IVC filter. 

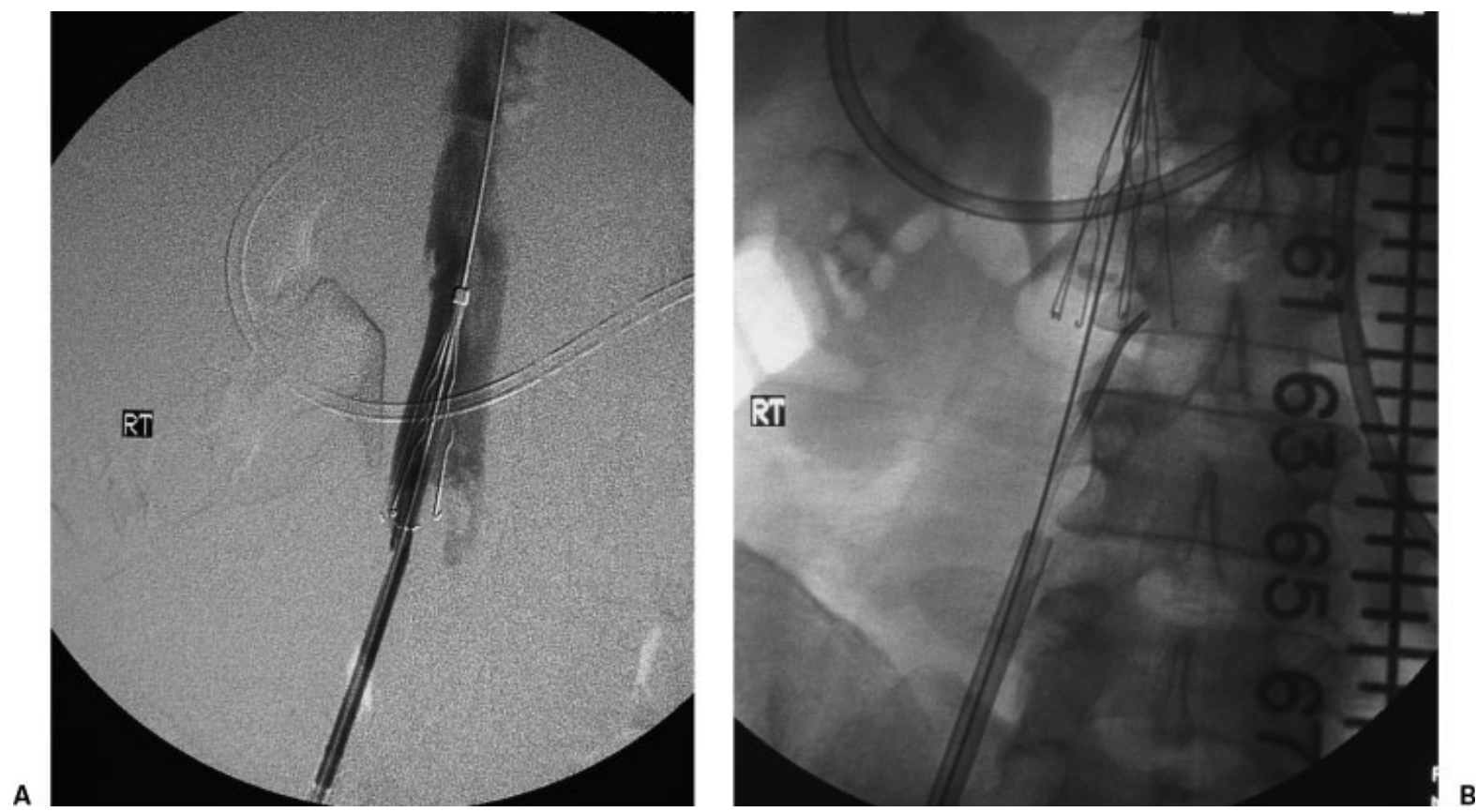

Figure 3 The patient had lower extremity DVT and a contraindication to OAT. A stainless steel Greenfield over-the-wire IVC filter was deployed and a postdeployment cavagram was done (A), which showed that the IVC filter did not cover the entire IVC width. Keeping the centering wire in place to stabilize the IVC filter, a Kumpe catheter was advanced up through the filter deployment sheath to manipulate the filter struts (B). A completion IVC study after IVC filter manipulation showed complete coverage of the IVC. An alternative, more conservative approach would include adding a second IVC filter.

as virtually all important caval anomalies and IVC filter insertion planning information is available on these studies. Very rarely a filter may fail to deploy properly or may be tilted with regard to the axis of the IVC. In the first instance the operator has to determine if the filter will provide the patient with adequate protection from $\mathrm{PE}$ and whether the filter is secure in position. Our group has advocated gentle manipulation in certain cases (Fig. 3), although this is counter to many of the instructions for use for permanent type IVC filters. ${ }^{33}$ The optional filters can be removed, the situation assessed, and another filter can be inserted. A last resort is to deploy a second filter more centrally to the compromised filter. The issue of tilted and asymmetric filters is controversial, with some investigators reporting no influence while others worry this compromises filter function (clot trapping and caval thrombosis). ${ }^{34,35}$

A related mechanical failure method involves a patient with an IVC filter who returns with symptoms of recurrent PE. ${ }^{4,36}$ Unfortunately for many patients, clinicians assume that a patient with an IVC filter in place is permanently protected from recurrent $\mathrm{PE}$. This misconception frequently delays the important evaluation of the IVC filter to ascertain if it is compromised in any fashion. Recurrent PEs after IVC filter insertion have a wide diversity of causes that should be considered in the workup. It is important to recognize that the filter itself may be a source of emboli if thrombus extends downstream from the top of the filter (Fig. 4A,B). Rarer causes include thrombosis of the filter with prominent collateral formation around the IVC filter or a migrated filter (Fig. 4C). Filter migration to the heart of pulmonary circulation may warrant emergent endovascular or surgical means to remove the migrated filter, and a second IVC filter may be required to protect the patent from additional emboli if the patient cannot be anticoagulated (Fig. 4D). Other instances of mechanical filter problems include filter strut fracture and guide wire entrapment. Strut fracture has not been reported to compromise filter function but it is conceivable that this could become an issue. Guide wire entrapment may compromise an IVC filter if the filter is pulled from its initial satisfactory position to another location, leaving the patient unprotected. If the filter is compromised and the patient cannot be anticoagulated, another filter may be required immediately depending upon clinical circumstances. Attempts at removing the attached guide wire are suggested. If the patient can be anticoagulated there may be a delay before the attempt at removing the attached guide wire. If the filter is still indicated and the original filter is compromised, a new filter will have to be inserted at another location, or if the compromised filter can be removed, another can be placed in the original location.

\section{Urgent Filter Placement/Intervention}

All patients with documented proximal DVT (popliteal and above) or with propagating distal DVT and a 

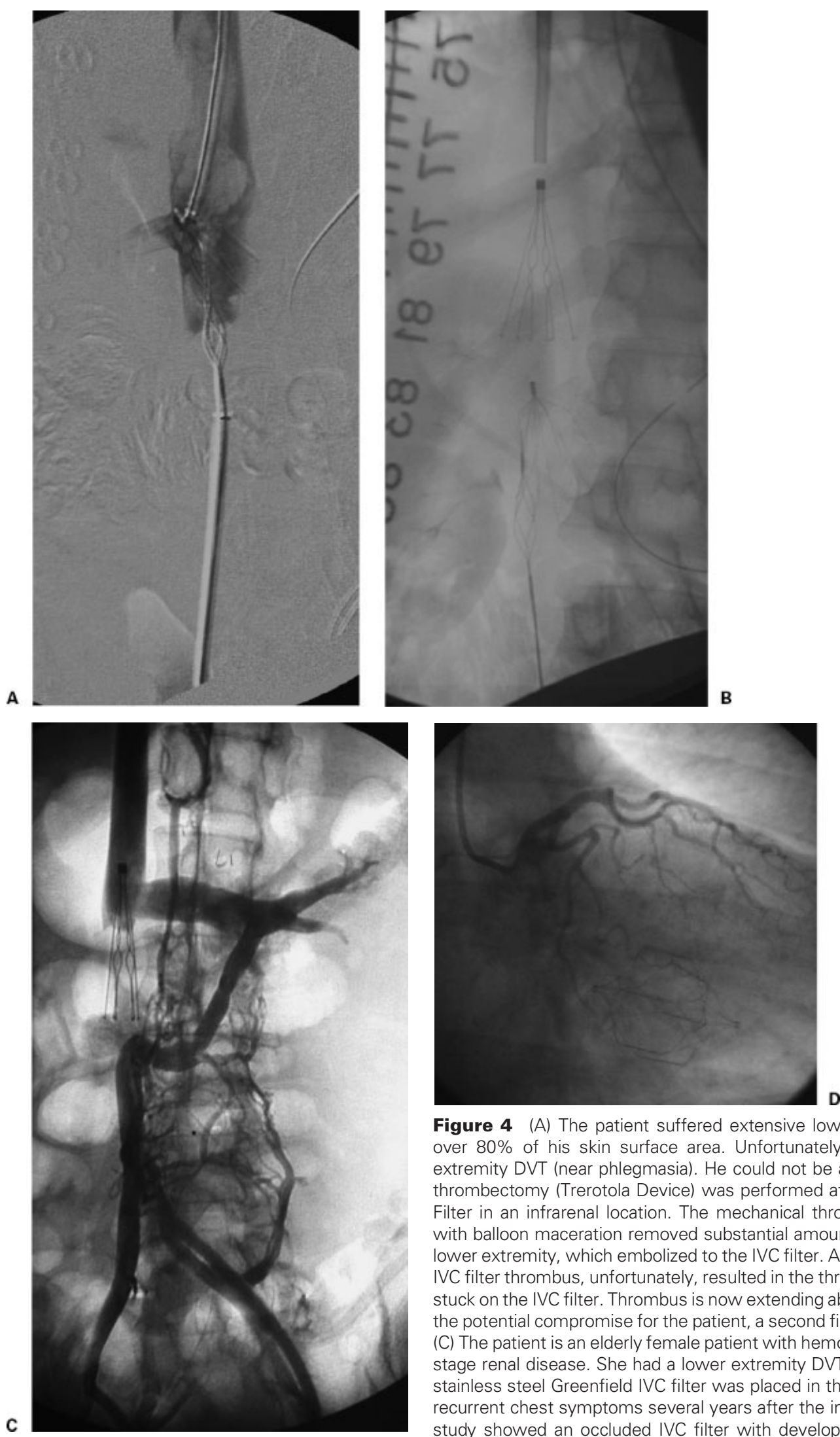

Figure 4 (A) The patient suffered extensive lower extremity and body burns over $80 \%$ of his skin surface area. Unfortunately, he developed a left lower extremity DVT (near phlegmasia). He could not be anticoagulated so mechanical thrombectomy (Trerotola Device) was performed after placement of a Recovery Filter in an infrarenal location. The mechanical thrombectomy device combined with balloon maceration removed substantial amounts of thrombus from the left lower extremity, which embolized to the IVC filter. An attempt to treat the IVC and IVC filter thrombus, unfortunately, resulted in the thrombectomy device becoming stuck on the IVC filter. Thrombus is now extending above the IVC filter. Because of the potential compromise for the patient, a second filter was placed above this (B). (C) The patient is an elderly female patient with hemodialysis catheter-treated endstage renal disease. She had a lower extremity DVT and bled while on OAT so a stainless steel Greenfield IVC filter was placed in the infrarenal position. She had recurrent chest symptoms several years after the initial filter placement. The IVC study showed an occluded IVC filter with development of prominent left renal collaterals (circumaortic renal vein [lower moiety] and prerenal left renal vein in conventional position [upper moiety]). A second filter was placed in a suprarenal location. (D) An elderly patient with renal cancer and lower extremity DVT had a Trapease IVC filter placed in an infrarenal location. He returned with symptoms of recurrent PE and also arrythmias. During his workup, a coronary angiogram revealed a migrated filter located in the right ventricle, which was removed surgically. He was treated with OAT. 
contraindication to OAT should undergo IVC filter placement. Because the risk of PE is spread over several days or weeks, ${ }^{22}$ the filter can be inserted on an urgent basis (between 24 and 48 hours) or sooner if possible. As mentioned above, if the patient has a large free-floating iliofemoral thrombus of greater than $5 \mathrm{~cm}$, emergent IVC filter insertion should be considered. In high-risk patients who require urgent surgery and have temporary or transient contraindications for OAT, filters may be inserted. These can include patients undergoing surgeries in which postoperative DVT may occur in upward of $25 \%$ of patients and in patients with added risk factors such as age $>40$ years, congestive heart failure, malignancy, obesity, previous DVT, and extended immobilization. ${ }^{21}$

If a patient who has been receiving OAT for a documented VTE develops a complication that requires premature discontinuation of this therapy, the patient should be considered high risk for another VTE event. The risk of recurrence varies with type of initial VTE event (PE or DVT) and the duration the patient was anticoagulated. In a patient in whom a complication develops shortly after initiation of OAT, a risk of PE comparable to those with an initial contraindication can be expected, and filters should be inserted in those patients according to their clinical presentation and status (see above). In other patients, complications may occur after several months of therapy with OAT. Because the majority of VTE recurrences occur within 3 months, OAT therapy can usually be stopped without additional protection. However, if multiple previous VTE events have been documented in that patient or the complication occurs within an intermediate time period ( 3 weeks to 3 months) since the initial event, the decision to filter or not needs to be based upon individual patient issues. For instance, if the OAT patient had initially presented with $\mathrm{PE}$ or has had multiple prior PEs and then develops a complication of OAT, then a filter should probably be inserted. In contrast, a patient who never sustained a PE but has received OAT for DVT can be followed up with noninvasive venous exams. If studies show active recurrent or propagating DVT, then a filter should be placed. Patients whose exams are normal can simply be followed up for the duration of the risk period. The current generation of retrievable filters allows much individualization to be applied in such cases.

Prophylactic IVC filters for patients at high risk for PE have been advocated, particularly for critically ill surgical or trauma patients. ${ }^{37}$ The average incidence of DVT in the general trauma population is $42 \%$ (ranging from 18 to 90\%) and the reported incidence of $\mathrm{PE}$ in patients with spinal cord injury is $10 \%$ (range 4 to $22 \%$ ). Prophylactic IVC filters should be placed in the following high-risk trauma patients with contraindications to anticoagulation $^{1}$ : age $>55$ years with isolated long bone fracture $^{2}$; severe head injury with coma $^{3}$; head injury with long bone fracture ${ }^{4}$; spinal cord injury with paraplegia or quadriplegia ${ }^{5}$; multiple long bone fractures with pelvic fracture ${ }^{6}$; multiple (four or more) long bone fractures ${ }^{7}$; and penetrating pelvic venous injury. In general, the IVC filters should be placed as soon after injury as possible and retrievable IVC filters should be considered when the risk of $\mathrm{PE}$ or contraindication to anticoagulation is anticipated to be short-term (weeks).

The use of IVC filters during thrombolysis of iliocaval venous thrombus is a controversial subject. Thrombolysis of iliocaval thrombi has been associated with a significant incidence of fatal PE (up to 6\%) by some investigators ${ }^{38,39}$ but not reported by others. ${ }^{40}$ Although the data are predominately observational, the evidence suggests that there is a real risk of $\mathrm{PE}$ associated with thrombolysis of central venous thrombi, particularly caval thrombi. Retrievable IVC filters would appear ideally suited for such applications.

\section{Elective Filter Placement/Intervention}

When patients with a history of significant VTE events after surgical procedures associated with high risk for $\mathrm{PE}$ and/or DVT, such as elective hip surgery, are scheduled for similar procedures, elective IVC filter placement can be considered to protect the patient during the period of time while the patient cannot be anticoagulated or is not able to ambulate. Again, retrievable IVC filters would likely be perfectly suited for such applications. Other investigational areas for elective application of IVC filters would include patients undergoing bariatric, neurosurgical, and orthopedic procedures. Patients who have been diagnosed with chronic $\mathrm{PE}$ and are being considered for pulmonary thromboendarterectomy are usually considered for elective insertion of IVC filters to prevent recurrences postoperatively. ${ }^{41}$ The removal of retrievable IVC filters is generally an elective procedure unless a complication such as filter migration has occurred.

\section{CONCLUSIONS}

IVC filters do appear to prevent PE, but in certain cases may be associated with excessive recurrent DVT and IVC filter thrombosis. The application of retrievable IVC filters is an attempt to shift the risk-benefit profile of IVC filters into an improved sector. Although the indications for IVC filters appear to increase with time because of wider application, it is best to try to adhere to strict criteria in situations were the benefits and risks are clearly defined and balanced toward patient benefit. The timing of filter insertion is not well detailed and is controversial; hopefully future studies will help define the parameters to follow. In the meantime using generalized guidelines and clinical judgment are warranted. 


\section{REFERENCES}

1. White RH. The epidemiology of venous thromboembolism. Circulation 2003;107:I4-I8

2. Barritt DW, Jordan SC. Anticoagulant drugs in treatment of pulmonary embolisms: a controlled clinical trail. Lancet 1960; 1:1309-1312

3. Levine M, Raskob GE, Landefeld CS, Kearnon C. Hemorrhage complications after anticoagulation treatment. Chest 1998;114(suppl):511S-523S

4. Kinney TB. Update on inferior vena cava filters. J Vasc Interv Radiol 2003;14:425-430

5. Greenfield LJ. Evolution of venous interruption for pulmonary thromboembolism. Arch Surg 1992;127:622-626

6. Greenfield LJ, McCrudy JR, Brown PP, Elkins RS. A new intracaval filter permitting continued flow and resolution of emboli. Surgery 1973;73:599-606

7. Becker DM, Philbrick JT, Selby JB. Inferior vena cava filters. Arch Intern Med 1992;152:1985-1994

8. Streiff MB. Vena caval filters: a comprehensive review. Blood 2000;95:3669-3677

9. Girard P, Stern JP, Parent F. Medical literature and vena cava filters: so far so weak. Chest 2002;122:963-967

10. Brender E. Use of emboli-blocking filters increases, but rigorous data are lacking. JAMA 2006;295:989-990

11. Decousus H, Leizorovicz A, Parent A, et al. A clinical trial of vena caval filters in the prevention of pulmonary embolism in patients with proximal deep-vein thrombosis. Prévention Du Risque D'Embolie Pulmonaire Par Interruption Cave Study group. N Engl J Med 1998;338:409-415

12. The PREPIC study group. Eight year follow-up of patients with permanent vena cava filters in prevention of pulmonary embolism. The PREPIC (Prévention Du Risque D'Embolie Pulmonaire par Interruption Cave) Randomized Study. Circulation 2005;112:416-422

13. Girard P, Musset D, Parent F, Maitre S, Philippoteau C, Simonneau G. High prevalence of detectable deep venous thrombosis in patients with acute pulmonary embolism. Chest 1999;116:903-908

14. Hyers TM. Management of venous thromboembolism: past, present, and future. Arch Intern Med 2003;163:759-768

15. Barritt DW, Jordan SC. Anticoagulant drugs in treatment of pulmonary embolism: a controlled trial. Lancet 1960;1:13091312

16. Cundiff DK, Manyemba J, Pezzullo JC. Anticoagulants versus non-steroidal anti-inflammatories or placebo for treatment of venous thromboembolism. Cochrane Database Syst Rev 2006;(1):CD003746

17. Douketis JD, Kearon C, Bates S, et al. Risk of fatal pulmonary embolism in patients with treated venous thromboembolism. JAMA 1998;279:458-462

18. Heit JA. Venous thromboembolism: disease burden, outcomes and risk factors. J Thromb Haemost 2005;3:1611-1617

19. Palareti G, Manotti C, D'Angelo A, et al. Thrombotic events during oral anticoagulation treatment: results of inceptioncohort, prospective, collaborative ISCOAT study. Thromb Haemost 1997;78:1438-1443

20. Kaufman J, Kinney TB, Streiff MB, et al. Guidelines for the use of retrievable and convertible vena cava filters: report for the SIR multidisciplinary consensus conference. J Vasc Interv Radiol 2006;17:449-459

21. Jones TK, Barnes RW, Greenfield LJ. Greenfield vena caval filter: rationale and current indications. Ann Thorac Surg 1986;42(suppl):S48-S55
22. Barker NW, Nygaard KK, Walters W, Priestly JT. A statistical study of postoperative venous thrombosis and pulmonary embolism: III. Time of occurrence during the post operative period. Proc Staff Mayo Clin 1941;16:17-21

23. Norris CS, Greenfield LJ, Herrmann JB. Free-floating iliofemoral thrombus. Arch Surg 1985;120:806-808

24. Pacouret G, Alison D, Pottier JM, Bertrand P, Charbonnier B. Free-floating thrombus and embolic risk with angiographically confirmed proximal deep venous thrombus: a prospective study. Arch Intern Med 1997;157:305-308

25. Dalen JE, Alpert JS. Natural history of pulmonary embolism. Prog Cardiovasc Dis 1975;17:259-270

26. Greenfield LJ, Peyton MD, Brown PP, Elkins RC. Transvenous management of pulmonary embolic disease. Ann Surg 1974;180:461-468

27. The urokinase pulmonary embolism trial: a national cooperative study. Circulation 1973;47(suppl 2):II1-II108

28. Greenfield LJ, Scher LA, Elkins RC. KMA-Greenfield filter placement for chronic pulmonary hypertension. Ann Surg 1979;189:560-565

29. Peyton JW, Hylemon MB, Greenfield LJ, et al. Comparison of Greenfield filter and vena cava ligation for experimental septic thromboembolism. Surgery 1983;93:533-537

30. Greenfield LJ, Proctor MC. Vena cava filter use in patients with sepsis: results in 175 patients. Arch Surg 2003;138: 1245-1248

31. Shimizu M, Tatsumi K, Matsukawa R, Shima T, Miwa Y. Retrievable Günther Tulip filter complicated by sepsis and retroperitoneal hemorrhage: successful management by filter retrieval. Intern Med 2005;44:593-597

32. Antevil JL, Sise MJ, Sack DI, et al. Retrievable vena cava filters for preventing pulmonary embolism in trauma patients: a cautionary tale. J Trauma 2006;60:35-40

33. Moore BS, Valji K, Roberts AC, Bookstein JJ. Transcatheter manipulation of asymmetrically opened Greenfield filters. J Vasc Interv Radiol 1993;4:687-690

34. Greenfield LJ, Proctor MC. Experimental embolic capture by asymmetric Greenfield filters. J Vasc Surg 1992;16:436465

35. Kinney TB, Rose SC, Weingarten KE, et al. IVC filter tilt and asymmetry: comparison of the over-the-wire stainless steel and titanium Greenfield IVC filters. J Vasc Interv Radiol 1997;8:1029-1037

36. David W, Gross WS, Colaiuta E, Gonda R, Osher D, Launti S. Pulmonary embolus after vena cava filter placement. Am Surg 1999;65:341-346

37. Rogers FB, Cipolle MD, Velmahos G, Royzyki G, Luchette FA. Practice management guidelines for prevention of venous thromboembolism in trauma patients: the EAST practice management guidelines work group. JTrauma 2002;53:142-164

38. Lorch H, Zwaan M, Siemens HJ, Wagner T, Kagel C, Weiss HD. Temporary vena cava filters and ultrahigh streptokinase thrombolysis therapy: a clinical study. Cardiovasc Intervent Radiol 2000;23:273-278

39. Grimm W, Schwieder G, Wagner T. Fatal pulmonary embolism in venous thrombosis of the leg and pelvis during lysis therapy. Dtsch Med Wochenschr 1990;115:1183-1187

40. Semba CP, Cake MD. Iliofemoral deep venous thrombosis: aggressive therapy with catheter-directed thrombolysis. Radiology 1994;191:487-494

41. Jamieson SW, Nomura K. Indications for and the results of pulmonary thromboendarterectomy for thromboembolic pulmonary hypertension. Semin Vasc Surg 2000;13:236-244 